\title{
SEVERAL INTERESTING INTEGRAL INEQUALITIES
}

\section{Wenjun LiU, QuốC-AnH NGô And Vu NHAT HuY}

Abstract. In this paper, several interesting integral inequalities are presented and some open problems are proposed later on.

Mathematics subject classification (2000): 26D15.

Keywords and phrases: integral inequality, Qi's inequality.

\section{REFERENCES}

[1] M. Ak Kouchi, Some integral inequalities, Divulgaciones Math., 11, 2 (2003), 121-125.

[2] M. AKKouchi, On an integral inequality of Feng Qi, Divulgaciones Math., 13, 1 (2005), 11-19.

[3] K. BRAhIM, On some q-integral inequalities, JIPAM. J. Inequal. Pure Appl. Math., 9, 4 (2008), Art. 106.

[4] L. Bougoffa, Notes on Qi type integral inequalities, JIPAM. J. Inequal. Pure Appl. Math., 4, 4 (2003), Art. 77.

[5] L. Bougoffa, An integral inequality similar to Qi's inequality, JIPAM. J. Inequal. Pure Appl. Math., 6, 1 (2005), Art. 27.

[6] K. BOUKERRIOUA AND A. GueZANE-LAKOUd, On an open question regarding an integral inequality, JIPAM. J. Inequal. Pure Appl. Math., 8, 3 (2007), Art. 77.

[7] L. Bougoffa, Note on an open problem, JIPAM. J. Inequal. Pure Appl. Math., 8, 2 (2007), Art. 58.

[8] Y. Chen And J. Kimball, Note on an open problem of Feng Qi, JIPAM. J. Inequal. Pure Appl. Math., 7, 1 (2005), Art. 4.

[9] B. Gavrea, On some integral inequalities, International Series of Numerical Mathematics 157, Birkhäuser Basel (2009), pp. 91-95.

[10] N.S. HoAng, Notes on an inequality, JIPAM. J. Inequal. Pure Appl. Math., 9, 2 (2008), Art. 42.

[11] The 2nd International Mathematical Competition for University Students, Plovdiv, Bulgaria, 2-7 August, 1995, Solutions for problems on Day 1, Available online at

URL: http: / / www. imc-math.org.uk/index.php?year=1995 or URL: http://www.ucl.ac.uk/ ucahjej/imc/imc1995/prob_sol.pdf.

[12] J.-Ch. KuAng, Applied inequalities, 3nd edition, Shandong Science and Technology Press, Jinan, China, 2004. (Chinese).

[13] W.J. LiU, C.C. LI AND J.W. Dong, Note on Qi's inequality and Bougoffa's inequality, JIPAM. J. Inequal. Pure Appl. Math., 7, 4 (2006), Art. 129.

[14] W.J. LiU, C.C. Li AND J.W. Dong, Consolidations of extended Qi's inequality and Bougoffa's inequality, J. Math. Inequal., 2, 1 (2008), 9-15.

[15] W.J. LiU, C.C. LI AND J.W. Dong, On an open problem concerning an integral inequality, JIPAM. J. Inequal. Pure Appl. Math., 8, 3 (2007), Art. 74.

[16] W.J. LIU, G.S. CHENG AND C.C. LI, Further development of an open problem concerning an integral inequality, JIPAM. J. Inequal. Pure Appl. Math., 9, 1 (2008), Art.14.

[17] Mathematics today 10, A.Ya. Dorogovtsev (Ed.), Nauchnoe Izdatel'stvo “TViMS”, Kiev, 1995, pp. 102.

[18] Y. MiAO AND J. Li, Further development of an open problem, JIPAM. J. Inequal. Pure Appl. Math., 9, 4 (2008), Art. 108.

[19] S. MAZOUZI AND F. QI, On an open problem regarding an integral inequality, JIPAM. J. Inequal. Pure Appl. Math., 4, 2 (2003), Art. 31. 
[20] Q.A. NGô, D.D. Thang, T. T. DAT AND D.A. TuAn, Note on an integral inequality, JIPAM. J. Inequal. Pure Appl. Math., 7, 4 (2006), Art. 120.

[21] T.K. PogAnY, On an open problem of F. Qi, JIPAM. J. Inequal. Pure Appl. Math., 3, 4 (2002), Art. 54.

[22] F. QI, Several integral inequalities, JIPAM. J. Inequal. Pure Appl. Math., 1, 2 (2000), Art. 19.

[23] F. QI, A.J. LI, W.Z. ZHAO, D.W. NIU, AND J. CAO, Extensions of several integral inequalities, JIPAM. J. Inequal. Pure Appl. Math., 7, 3 (2006), Art. 107.

[24] W. T. Sulaiman, On an integral inequality, Commun. Math. Anal., 5, 1 (2008), 57-64.

[25] K.W. YU AND F. QI, A short note on an integral inequality, RGMIA Res. Rep. Coll., 4, 1 (2001), Art. $4,23-25$. 